Author affiliations and support information (if applicable) appear at the end of this article.

Published at jco.org on April 20, 2017.

Corresponding author: Michael D'Angelica, MD, FACS, Memorial Sloan Kettering Cancer Center, Department of Surgery, 1275 York Avenue, New York, NY 10065; dangelim@mskcc.org.

(C) 2017 by American Society of Clinical Oncology

$0732-183 X / 17 / 3517 w-1938 w / \$ 20.00$

\title{
Perioperative Hepatic Arterial Infusion Pump Chemotherapy Is Associated With Longer Survival After Resection of Colorectal Liver Metastases: A Propensity Score Analysis
}

Bas Groot Koerkamp, Eran Sadot, Nancy E. Kemeny, Mithat Gönen, Julie N. Leal, Peter J. Allen, Andrea Cercek, Ronald P. DeMatteo, T. Peter Kingham, William R. Jarnagin, and Michael I. D'Angelica

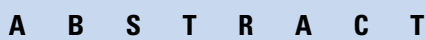

\section{Purpose}

To investigate whether perioperative hepatic arterial infusion pump chemotherapy (HAl) was associated with overall survival (OS) in patients who had a complete resection of colorectal liver metastases (CLM).

\section{Methods}

Patients who underwent a complete resection of CLM between 1992 and 2012 were included from a single-center prospectively maintained database. All patients who received $\mathrm{HAl}$ also received perioperative systemic chemotherapy. Propensity score analysis was used to match patients for seven known prognostic factors.

\section{Results}

A total of 2,368 consecutive patients underwent a complete resection of CLM, with a median followup of 55 months. The median OS for patients with HAI $(n=785)$ was 67 months versus 44 months without HAI $(n=1,583 ; P<.001)$, despite more advanced disease in the HAl group. OS at 10 years was $38.0 \%$ versus $23.8 \%$ without $\mathrm{HAl}$. For patients who received modern systemic chemotherapy ( $n=1,442$ ), the median OS was 67 months with $\mathrm{HAl}$ and 47 months without $\mathrm{HAl}(P<.001)$. The hazard ratio adjusted by propensity score demonstrated longer OS with $\mathrm{HAl}: 0.67(95 \% \mathrm{Cl}, 0.59$ to 0.76; $P<.001)$. A pronounced difference in median OS was found for patients with node-negative colorectal cancer (129 months with HAl $v 51$ months without; $P<.001$ ) and a low clinical risk score of 0 to 2 points (89 months with HAl $v 53$ months without; $P<.001$ ).

\section{Conclusion}

Patients who received HAI had a median OS of approximately 2 years longer than patients without $\mathrm{HAl}$. The strong association was independent of the use of modern systemic chemotherapy and remained in propensity score analysis. Patients with node-negative primary tumors or a low clinical risk score seemed to benefit most from HAl.

\section{J Clin Oncol 35:1938-1944. (C) 2017 by American Society of Clinical Oncology}

\section{INTRODUCTION}

Colorectal cancer is the third most common cancer in the United States, with an annual incidence of 137,000 patients. The majority of these patients present with or eventually develop colorectal liver metastases (CLM). ${ }^{1}$ Patients with resectable CLM have a 10 -year overall survival (OS) of approximately 20\%. ${ }^{2}$ Unfortunately, most patients develop recurrent disease after resection. ${ }^{2}$ Perioperative systemic chemotherapy is generally recommended to avert or delay recurrence, although randomized controlled trials
(RCTs) have failed to demonstrate a difference in OS. ${ }^{3,4}$

Among the patients who develop a recurrence after resection of CLM, the liver is the only site of initial recurrence in approximately half of patients. ${ }^{5}$ Therefore, adjuvant regional chemotherapy administered in the hepatic artery has been investigated. The biologic rationale for hepatic arterial infusion pump chemotherapy (HAI) is that the hepatic artery, rather than the portal vein, provides most of the blood supply to CLM. ${ }^{6}$ Moreover, drugs such as floxuridine (FUDR) have a high first-pass extraction in the liver, allowing a high dosage in the liver with 
minimal systemic exposure. ${ }^{7}$ HAI can be administered via a surgically implantable subcutaneous pump with a catheter in the gastroduodenal artery. If HAI is used, systemic chemotherapy is also used. A phase III RCT at Memorial Sloan Kettering Cancer Center (MSKCC) found a 2 -year OS of $86 \%$ for patients who were treated with both systemic fluorouracil (FU) and HAI-FUDR compared with $72 \%$ with systemic $\mathrm{FU}$ alone after resection of CLM $(P=.03){ }^{8,9}$ A multicenter RCT found a statistically significant reduction in recurrence-free and hepatic recurrence-free survival after resection in patients treated with adjuvant HAI plus systemic chemotherapy as compared with surgery alone. ${ }^{10} \mathrm{~A}$ third multicenter RCT used HAI 5-FU instead of FUDR and was closed prematurely when no difference was found at interim analysis. ${ }^{11}$

The main criticism of these RCTs was that they were performed before the introduction of modern systemic chemotherapy such as oxaliplatin and irinotecan. Therefore, adjuvant HAI for CLM has not been widely adopted. We have continued to study HAI combined with modern systemic chemotherapy in phase I and II trials with favorable results. ${ }^{12-16}$

In the current study, we evaluated all consecutive patients who underwent complete resection of CLM during a 21-year period at a single center and compared patients with and without perioperative HAI. The first objective was to investigate whether perioperative HAI is associated with OS using propensity score analysis. The second objective was to characterize subgroups of patients who may benefit the most or least from HAI.

\section{METHODS}

\section{Patients}

From 1992 to 2012, consecutive patients who underwent a complete resection of CLM were included from a prospectively maintained liver resection database at MSKCC. Patients with macroscopic residual disease after resection or treated exclusively with ablation were excluded. Patients with unresectable CLM at presentation were included if they eventually had a complete resection of CLM after downstaging. Patients with completely resected extrahepatic disease (EHD) diagnosed before or at the time of liver resection, as well as patients with postoperative mortality, were included. The study was performed after approval by the Institutional Review Board of MSKCC.

\section{Variables}

Clinical and pathologic data were collected prospectively. Disease-free interval was defined as the period from resection of the primary colorectal cancer to diagnosis of CLM. When CLM were diagnosed at the time of resection of the primary tumor they were classified as synchronous. $\mathrm{Pa}$ tients with node-positive primary tumors were further classified as N1 or N2 according to the seventh edition of the American Joint Committee on Cancer staging. ${ }^{17}$ The number and maximal diameter of CLM were derived from the pathology report. Tumors with a complete pathologic response were included in the total tumor count. Tumors ablated at the time of curative-intent resection were also added to the total tumor count. The serum carcinoembryonic antigen level was collected as the highest value in the 3 months before resection. These five prognostic factors were combined in the clinical risk score (CRS) as described previously. ${ }^{18}$ Patients were further subdivided on the basis of the CRS into low risk ( 0 to 2 points) and high risk ( 3 to 5 points). The closest hepatic resection margin was measured down to tenths of a millimeter. A positive margin was defined as the presence of tumor cells at the surgical resection margin. EHD was defined as any completely resected colorectal cancer outside the liver diagnosed before or at the time of liver resection.

\section{Work-Up and Treatment}

Preoperative imaging included cross-sectional imaging with contrastenhanced computed tomography (CT) and/or magnetic resonance imaging of the abdomen and pelvis and CT of the chest. Fluorodeoxyglucose-positron emission tomography (PET) scans were used selectively at the discretion of the treating physicians. Intraoperative ultrasound was routinely performed. Lymphadenectomy of the hepatoduodenal ligament was performed selectively when lymph nodes appeared suspicious on imaging or intraoperatively. ${ }^{19}$ Two-stage resections were performed as previously described. ${ }^{20}$

Systemic chemotherapy regimens varied over time and were determined by the treating medical oncologist on the basis of guidelines, previous chemotherapy history, and ongoing clinical trials. Modern chemotherapy was defined as regimens containing oxaliplatin or irinotecan. Adjuvant systemic chemotherapy was defined as systemic chemotherapy starting within 3 months after resection of CLM. Preoperative systemic chemotherapy was defined as systemic chemotherapy within a 3month period before resection of CLM.

All patients receiving HAI were scheduled to receive 5-FU with or without additional systemic chemotherapy such as irinotecan or oxaliplatin. ${ }^{8}$ A switch to systemic chemotherapy alone only happened when the patient could no longer tolerate HAI. Patients were selected for HAI in the setting of clinical trials or outside trials at the discretion of the treating physicians. The implantable pump was placed at the time of resection and positioned subcutaneously in the abdominal wall, with the catheter tip typically positioned in the gastroduodenal artery as previously reported. ${ }^{8}$ Bilobar hepatic perfusion and lack of extrahepatic perfusion were confirmed by both intraoperative dye testing and postoperative technetium99-labeled macroaggregated albumin nuclear medicine scanning. Patients with extrahepatic perfusion were evaluated angiographically and aberrant branches embolized with retesting before treatment. Patients received intra-arterial FUDR, with six cycles scheduled. Patients who did not complete all six cycles or who never received HAI because of technical failure remained in the HAI group for all analyses. HAI was mostly administered in the adjuvant setting after complete resection of CLM but was also administered preoperatively for patients with unresectable disease at presentation. ${ }^{12}$ Patients who had a pump placed for HAI at the time of recurrence after resection of CLM but did not receive perioperative HAI at the time of the initial hepatic resection were analyzed in the no-HAI group. The pump was typically removed after 2 years follow-up. A pump was rarely removed earlier unless it caused substantial discomfort to the patient or if the pump became nonfunctional.

\section{Statistical Analyses}

Propensity score analysis was performed using seven known independent prognostic factors. ${ }^{21}$ Quartiles of the propensity score were estimated using sex, age, year of resection, presence of extrahepatic disease, number of resected or ablated tumors, size of largest resected tumor, and margin status. All continuous prognostic factors (eg, age) were used as continuous variables. Imputation was not required for propensity score analysis, because the data were $98.5 \%$ complete for all seven covariates.

Prognostic factors between patients with and without HAI were compared using the Fisher's exact or $\chi^{2}$ test. OS from any cause was the outcome of interest for all analyses and was defined as survival in months from the date of the first resection of CLM to the date of death. Patients alive were censored at the date of last follow-up. Kaplan-Meier estimates and the log-rank test were used to compare the association between dichotomous and categorical prognostic factors and OS. Comparisons of median OS at 5 or 10 years were performed using the asymptotic results that complimentary log-transformation of Kaplan-Meier estimates are normally distributed and using a $z$-test to compare the two transformed survival probabilities. ${ }^{22}$ 
All analyses were performed using SPSS (IBM, version 22 for Mac) or $\mathrm{R}$ (version 3.0.2). A $P$ value $<.05$ was considered statistically significant.

\section{RESULTS}

\section{Patients}

Between 1992 and 2012, 4,915 patients underwent a liver resection at MSKCC, of whom 2,368 patients underwent a curative-intent resection of CLM. Forty-five patients (1.9\%) were excluded because of having macroscopic residual disease. Most patients $(2,193,92.7 \%)$ received preoperative and/or adjuvant systemic chemotherapy; 1,196 patients (50.5\%) received both preoperative and adjuvant systemic chemotherapy. A total of 1,583 patients $(66.8 \%)$ were treated without HAI, and 785 patients (33.2\%) were treated with HAI.

Table 1 compares baseline characteristics and systemic treatment between patients treated with and without HAI. Patients with HAI were more likely to have advanced disease, such as N2 disease, higher number of tumors, or synchronous CLM. Furthermore, the rates of two-stage resection and the use of intraoperative ablation were higher in the HAI group. Patients in the no-HAI group were older, with a higher rate of EHD. Patients receiving HAI were more often treated in the recent period (2003 to 2012) and were more likely to receive preoperative systemic chemotherapy.

\section{HAI}

HAI was administered in the adjuvant (ie, postoperative) setting in 653 patients $(83.2 \%)$ and in the preoperative setting in 132 patients $(16.8 \%)$. Of the 132 patients who received preoperative HAI, 79 (60\%) patients also received adjuvant HAI after complete resection of CLM.

All patients who received HAI also received preoperative and/or adjuvant systemic chemotherapy. Of the patients receiving HAI,

\begin{tabular}{|c|c|c|c|c|}
\hline Total & 2,368 & $1,583(66.8)$ & 785 (33.2) & - \\
\hline Male sex & $1,350(57.0)$ & $906(57.2)$ & $444(56.6)$ & .76 \\
\hline Period 2003-2012 & $1,295(55.7)$ & 767 (48.5) & $528(67.3)$ & $<.001$ \\
\hline $\mathrm{N}$ stage primary tumor & & & & .02 \\
\hline No & 908 (38.3) & 632 (39.9) & $276(35.2)$ & \\
\hline Synchronous & $1,181(49.9)$ & $713(45.0)$ & $468(59.6)$ & $<.001$ \\
\hline $\mathrm{DFI}<12$ months & $1,504(63.5)$ & $923(58.3)$ & $581(74.0)$ & $<.001$ \\
\hline No. of resected or ablated tumors & & & & $<.001$ \\
\hline Mean & 2.8 & 2.4 & 3.5 & \\
\hline 1 & $1,010(42.7)$ & $776(49.0)$ & $234(29.8)$ & \\
\hline 2 & 462 (19.5) & $332(21.0)$ & $130(16.6)$ & \\
\hline$>5 \mathrm{~cm}$ & $714(30.2)$ & $506(32.0)$ & $208(26.5)$ & .004 \\
\hline Mean, cm & 4.2 & 4.3 & 3.9 & \\
\hline $\mathrm{CEA}>200 \mathrm{ng} / \mathrm{ml}^{*}$ & $178(8.4)$ & $121(8.8)$ & $57(7.5)$ & .33 \\
\hline Clinical risk score* & & & & $<.001$ \\
\hline 0 & $67(3.2)$ & $55(4.0)$ & $12(1.6)$ & \\
\hline 1 & $390(18.4)$ & $298(21.8)$ & $92(12.3)$ & \\
\hline 2 & $672(31.8)$ & $440(32.2)$ & $232(31.0)$ & \\
\hline 3 & 747 (35.3) & 421 (30.8) & $326(43.5)$ & \\
\hline 4 & $196(9.3)$ & $125(9.1)$ & $71(9.5)$ & \\
\hline 5 & $44(2.1)$ & $28(2.0)$ & $16(2.1)$ & \\
\hline High CRS & $987(46.6)$ & $574(42.0)$ & $413(55.1)$ & $<.001$ \\
\hline Extrahepatic disease & $195(8.2)$ & $153(9.7)$ & $42(5.4)$ & $<.001$ \\
\hline Two-stage hepatectomy & $52(2.2)$ & $8(0.5)$ & $44(5.6)$ & $<.001$ \\
\hline
\end{tabular}


$87.1 \%$ received at least one cycle of perioperative modern systemic chemotherapy. Since 1998, this percentage has increased to $96.1 \%$. Nearly all patients $(129 ; 97.7 \%)$ who received preoperative HAI and systemic chemotherapy also received adjuvant systemic chemotherapy. Most patients (484; 74.1\%) who received adjuvant HAI chemotherapy also received preoperative systemic chemotherapy.

During follow-up, 127 patients (16.2\%) developed recurrent disease confined to the liver, of whom 63 patients $(49.6 \%)$ restarted HAI at the time of recurrence. An additional 48 patients were analyzed in the no-HAI group because they received a pump and HAI only at the time of recurrence after the initial resection of CLM.

\section{Overall Survival}

For the whole cohort, the estimated median OS was 51 months, with a 5 -year OS of $43 \%$, and a 10 -year OS of $29 \%$. During follow-up, 1,373 patients $(58 \%)$ died. The median followup of survivors was 55 months. The study population included 215 actual 10-year survivors. For patients who underwent a resection since 2003, the median OS was 57 months; 5-year OS was $48 \%$ and 10 -year OS was $37 \%$.

OS for patients treated with HAI was longer than patients without HAI (median OS, $67 v 44$ months, respectively; $P<.001$ ). Five-year OS for patients treated with HAI versus without HAI was $52.9 \%$ versus $37.9 \%$, respectively $(P<.001)$. Ten-year OS for patients treated with HAI versus without HAI was $38.0 \%$ versus $23.8 \%$, respectively $(P<.001)$. Median OS was longer for patients with HAI in both the early and late era (Table 2; Appendix Fig A1, online only).

\section{Poor Prognostic Factors}

Table 2 lists the results of univariate analyses of clinical and pathologic factors and OS. Poor prognostic factors included: age $>65$ years, resection before 2003, node-positive primary tumor, increased number of resected tumors, increased size of the largest resected tumor, preoperative carcinoembryonic antigen level $>200$, increased CRS, EHD, and positive resection margin. Both HAI and adjuvant modern systemic chemotherapy were associated with a favorable OS on univariate analysis.

The difference in OS between patients with and without HAI remained similar after excluding patients with EHD (median OS, $68 v 46$ months; $P<.001$ ). Figure 1 demonstrates that the difference in OS remained similar after excluding patients who did not receive modern systemic chemotherapy (median OS, $67 v$ 47 months; $P<.001)$.

\section{Propensity Score Analysis}

The hazard ratio (HR) adjusted by propensity score demonstrated longer OS associated with perioperative HAI (HR, 0.67; $95 \% \mathrm{CI}, 0.59$ to $0.76 ; P<.001$ ). Detailed results of propensity score analysis are listed in Appendix Table A1 (online only).

\section{Subgroup Analyses}

The differences in median OS between patients with and without HAI are listed in Table 2. HAI was associated with an improved OS in all subgroups except for patients with EHD, a positive resection margin, or a CRS of 4 or 5 . In general, the difference was smaller with more advanced disease, such as a CRS of 4 or 5 , or 10 or more tumors. Subgroups with a node-negative primary tumor or a low CRS ( 0 to 2 points) had a pronounced difference in median OS between those treated with and without HAI. Other subgroups with a difference in median OS between patients with and without HAI of $>24$ months included: age $>65$ years, metachronous tumors, disease-free interval of $>1$ year, and four or fewer tumors resected.

\section{DISCUSSION}

In this study with 2,368 consecutive patients who had a complete resection of CLM, we found that the median OS for patients treated with HAI was 67 months as compared with 44 months for those treated without HAI $(P<.001)$. In propensity score analysis, the same strong association with OS was found with an adjusted HR for HAI of $0.67(P<.001)$.

These results are consistent with our previous phase III trial. ${ }^{8,9}$ In the era of modern systemic chemotherapy, we performed a matched cohort analysis $(n=250)$ and found that adjuvant HAI was independently associated with improved disease-free survival (HR, 0.39; $P<.01) .{ }^{23}$ The current study confirms in a much larger and consecutive cohort that HAI is associated with improved OS (67 $v 47$ months; $P<.001)$ in patients $(\mathrm{n}=1,442)$ who received perioperative modern systemic chemotherapy.

OS for patients in this study without HAI was similar to OS in other large studies without HAI. For example, one of the largest published series on resected CLM is from two centers in the United Kingdom including 2,715 patients between 1987 and 2010. ${ }^{24}$ None of these patients received HAI. The median OS was 45 months, which is nearly identical to the 44 months in the no-HAI group $(\mathrm{n}=$ 1,583 ) of the current study. In contrast, the median OS in the HAI group $(n=785)$ of the current study was 67 months, even though these patients had more advanced disease (eg, 55\% high CRS).

In this study we also demonstrated that HAI is associated with improved OS in all subgroups except patients with EHD, a positive resection margin, or a CRS of 4 or 5 . In general, the improvement in OS with HAI was smaller with more advanced disease. Patients with node-negative colorectal cancer $(n=908)$ showed the largest difference in median OS: 129 months for patients with HAI and 51 months for patients without HAI $(P<.001)$. Patients with a low CRS $(\mathrm{n}=1,129)$ had a median OS of 89 months with HAI and 53 months without $(P<.001)$. Patients with fewer than five CLM also had better OS with HAI chemotherapy. These results are promising, because adjuvant systemic chemotherapy failed to prolong OS in patients with fewer than five CLM. ${ }^{3}$ We hypothesize that patients whose disease was less likely to progress outside the liver (eg, patients with a low CRS and no EHD) were more likely to benefit from perioperative HAI. Several subgroups (eg, N2 primary tumors) had a smaller but clinically relevant difference in OS with HAI. Patients who are unlikely to benefit from HAI (eg, patients with EHD) should be considered for systemic chemotherapy alone.

Other approaches for HAI used percutaneous catheter placement, a mediport, and other intra-arterial drugs such as oxaliplatin. ${ }^{25}$ The main challenges with percutaneous placement for intra-arterial FUDR is to avoid extrahepatic perfusion causing 
Groot Koerkamp et al

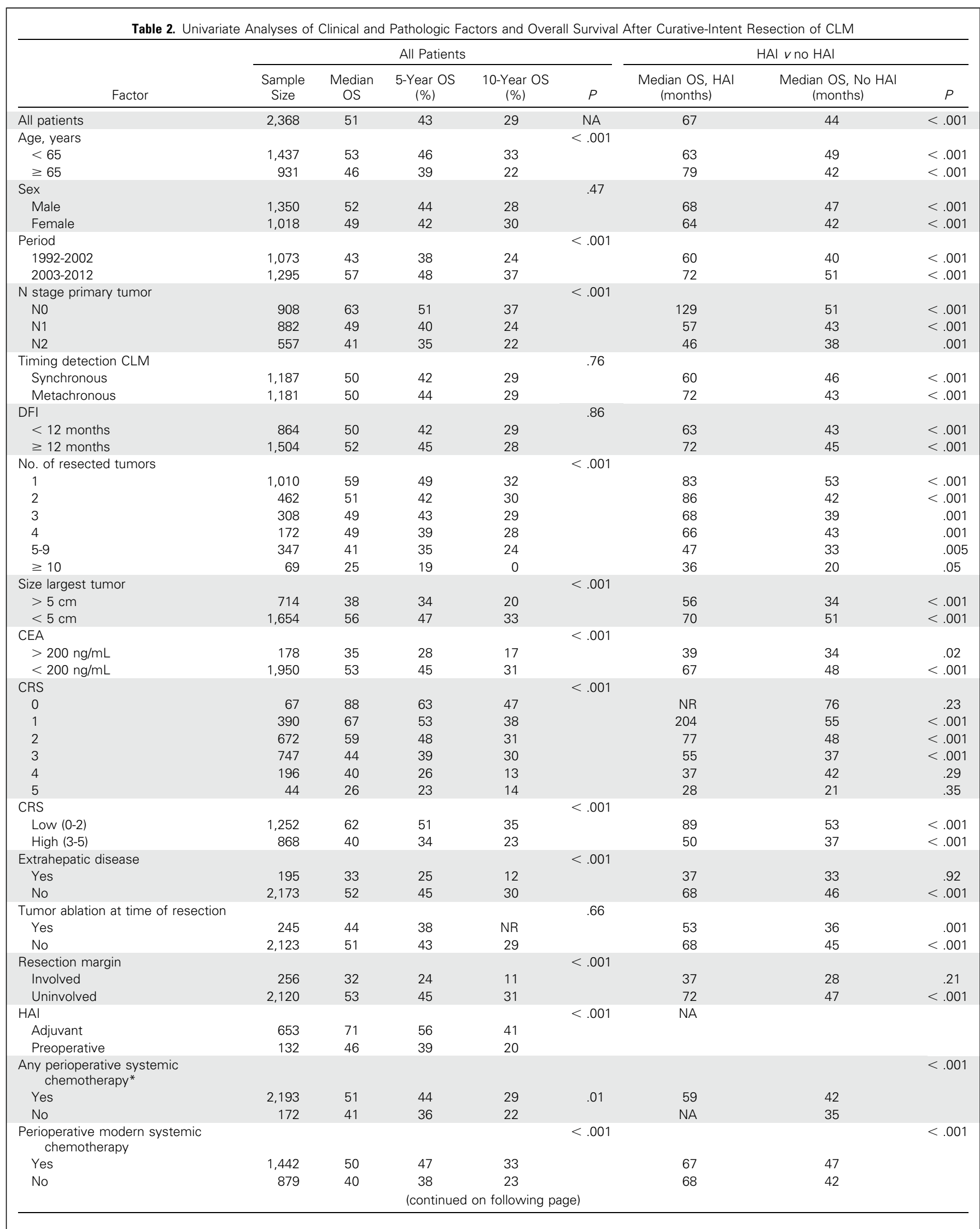




\begin{tabular}{|c|c|c|c|c|c|c|c|c|}
\hline \multirow[b]{2}{*}{ Factor } & \multicolumn{5}{|c|}{ All Patients } & \multicolumn{3}{|c|}{$\mathrm{HAl} v$ no HAl } \\
\hline & $\begin{array}{l}\text { Sample } \\
\text { Size }\end{array}$ & $\begin{array}{l}\text { Median } \\
\text { OS }\end{array}$ & $\begin{array}{c}5 \text {-Year OS } \\
(\%)\end{array}$ & $\begin{array}{c}\text { 10-Year OS } \\
(\%)\end{array}$ & $P$ & $\begin{array}{l}\text { Median OS, HAl } \\
\text { (months) }\end{array}$ & $\begin{array}{l}\text { Median OS, No HAI } \\
\text { (months) }\end{array}$ & $P$ \\
\hline $\begin{array}{l}\text { Preoperative modern systemic } \\
\text { chemotherapy }\end{array}$ & & & & & .99 & & & \\
\hline Yes & 812 & 51 & 43 & 29 & & 77 & 45 & $<.001$ \\
\hline No & 1,556 & 50 & 43 & 29 & & 55 & 43 & .004 \\
\hline $\begin{array}{l}\text { Adjuvant modern systemic } \\
\text { chemotherapy }\end{array}$ & & & & & $<.001$ & & & \\
\hline Yes & 1,124 & 57 & 48 & 35 & & 68 & 49 & $<.001$ \\
\hline No & 1,244 & 44 & 38 & 23 & & 59 & 41 & $<.001$ \\
\hline
\end{tabular}

severe ulcer disease and catheter dysfunction due to displacement or occlusion. Goéré et $\mathrm{al}^{26}$ investigated adjuvant HAI with oxaliplatin instead of FUDR in 44 patients with at least four CLM. They found a promising 3-year DFS of $75 \%$.

Critics of HAI have argued that the OS benefit in our RCT ${ }^{8}$ might disappear in the era of modern systemic chemotherapy. However, the current study demonstrates that the associated improvement in OS with HAI of approximately 2 years remained unchanged in the era of modern systemic chemotherapy. A reason for lack of dissemination of HAI is the complexity of HAI requiring multidisciplinary skills and experience. Surgical placement of the pump for HAI after completion of the liver resection requires an estimated learning curve of 25 procedures. ${ }^{27}$ Intensive management by the medical oncologist is required to preclude biliary

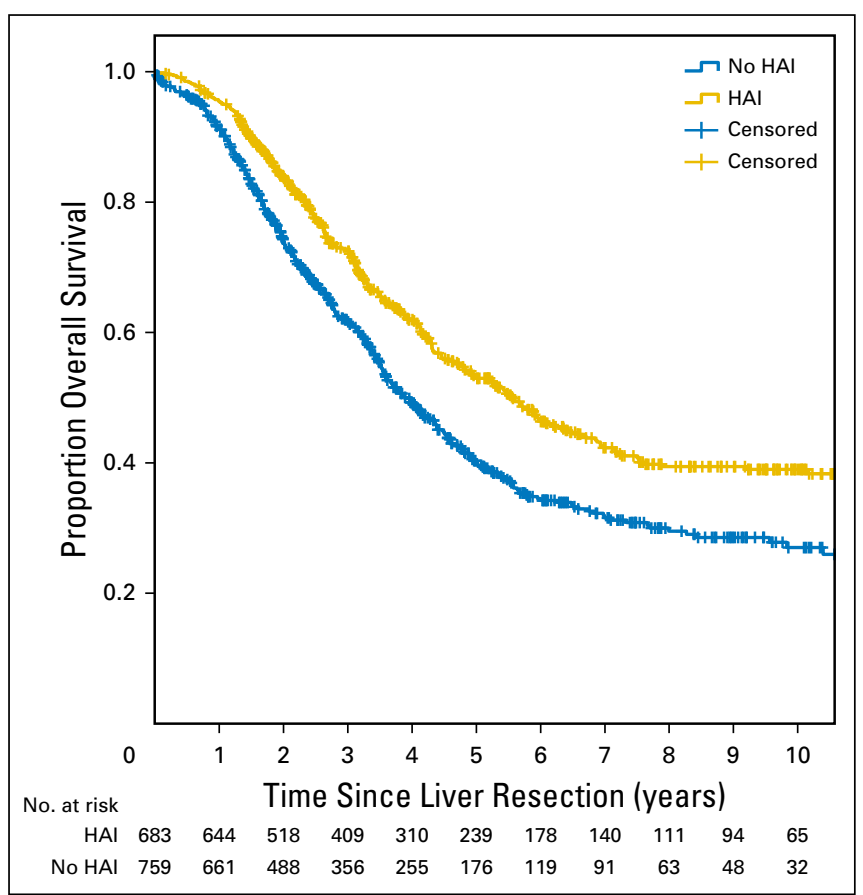

Fig 1. All patients underwent a curative-intent resection of colorectal liver metastases. Patients who did not receive modern systemic chemotherapy were excluded. The median overall survival was 67 months with hepatic arterial infusion pump chemotherapy (HAl) and 47 months without $\mathrm{HAI}(P<.001)$. toxicity. ${ }^{28}$ Nuclear medicine, interventional radiology, and gastroenterologists are involved in avoiding and managing complications of HAI (eg, bleeding or ulcer disease).

The current study has several limitations. Most importantly, the comparison between patients with and without HAI was nonrandomized, and patients were included over a 21-year period. Differences between treatment groups, however, were addressed with propensity score analysis, demonstrating an impressive HR of 0.67 after adjustment for seven independent poor prognostic factors, including year of resection. Unmeasured prognostic factors could be unbalanced across treatment groups and partly explain the difference in OS. However, patients receiving HAI had more advanced disease, considering the measured prognostic factors. Second, the study covers a period of 21 years including the introduction of irinotecan, oxaliplatin, and targeted treatments. The results of this study demonstrated that the favorable median OS after HAI remained in patients receiving modern systemic chemotherapy (Fig 1). Third, we did not have data on PET-CT aimed to improve preoperative staging. However, a recent study demonstrated that PET-CT before resection of CLM did not result in frequent change in surgical management. ${ }^{29}$

An RCT is needed to confirm that adjuvant HAI in the current era improves OS in patients with complete resection of CLM. The largest association between HAI and OS was found in this study for patients with node-negative colorectal cancer and patients with a low CRS. Confirmation of efficacy is most likely for these subgroups of patients. Future research should also evaluate genomic data (eg, KRAS status) to improve patient selection.

In conclusion, HAI was independently associated with an improvement in median OS of 23 months. This strong association remained in patients who received modern systemic chemotherapy and after adjustment for seven independent prognostic factors in propensity score analysis. Patients with a node-negative colorectal cancer or a low CRS seemed to benefit the most. An RCT is needed to confirm these findings.

\section{AUTHORS' DISCLOSURES OF POTENTIAL CONFLICTS OF INTEREST}

Disclosures provided by the authors are available with this article at jco.org. 


\section{AUTHOR CONTRIBUTIONS}

Conception and design: Bas Groot Koerkamp, Eran Sadot, Nancy E. Kemeny, Mithat Gönen, Peter J. Allen, Andrea Cercek, Ronald P. DeMatteo, T. Peter Kingham, William R. Jarnagin, Michael I. D’Angelica Administrative support: Nancy E. Kemeny, Michael I. D’Angelica Financial support: Bas Groot Koerkamp, William R. Jarnagin Provision of study materials or patients: Nancy E. Kemeny, Peter J. Allen, Andrea Cercek, Ronald P. DeMatteo, T. Peter Kingham, William R. Jarnagin, Michael I. D’Angelica
Collection and assembly of data: Bas Groot Koerkamp, Eran Sadot, Nancy E. Kemeny, Julie N. Leal

Data analysis and interpretation: Bas Groot Koerkamp, Eran Sadot, Nancy E. Kemeny, Mithat Gönen, Peter J. Allen, Ronald P. DeMatteo, T. Peter Kingham, William R. Jarnagin, Michael I. D’Angelica

Manuscript writing: All authors

Final approval of manuscript: All authors

Accountable for all aspects of the work: All authors

\section{REFERENCES}

1. Siegel R, Ma J, Zou Z, et al: Cancer statistics, 2014. CA Cancer J Clin 64:9-29, 2014

2. Tomlinson JS, Jarnagin WR, DeMatteo RP, et al: Actual 10-year survival after resection of colorectal liver metastases defines cure. J Clin Oncol 25:4575-4580, 2007

3. Nordlinger B, Sorbye $H$, Glimelius $B$, et al: Perioperative FOLFOX4 chemotherapy and surgery versus surgery alone for resectable liver metastases from colorectal cancer (EORTC 40983): Long-term results of a randomised, controlled, phase 3 trial. Lancet Oncol 14:1208-1215, 2013

4. Portier G, Elias D, Bouche O, et al: Multicenter randomized trial of adjuvant fluorouracil and folinic acid compared with surgery alone after resection of colorectal liver metastases: FFCD ACHBTH AURC 9002 trial. J Clin Oncol 24:4976-4982, 2006

5. de Jong MC, Pulitano $C$, Ribero $D$, et al: Rates and patterns of recurrence following curative intent surgery for colorectal liver metastasis: An international multi-institutional analysis of 1669 patients. Ann Surg 250:440-448, 2009

6. Ackerman NB: The blood supply of experimental liver metastases. IV. Changes in vascularity with increasing tumor growth. Surgery 75:589-596, 1974

7. Ensminger WD, Gyves JW: Clinical pharmacology of hepatic arterial chemotherapy. Semin Oncol 10:176-182, 1983

8. Kemeny N, Huang Y, Cohen AM, et al: Hepatic arterial infusion of chemotherapy after resection of hepatic metastases from colorectal cancer. N Engl J Med 341:2039-2048, 1999

9. Kemeny NE, Gonen M: Hepatic arterial infusion after liver resection. N Engl J Med 352:734-735, 2005

10. Kemeny MM, Adak S, Gray B, et al: Combinedmodality treatment for resectable metastatic colorectal carcinoma to the liver: surgical resection of hepatic metastases in combination with continuous infusion of chemotherapy-an intergroup study. J Clin Oncol 20:1499-1505, 2002
11. Lorenz $M$, Müller $H H$, Schramm $H$, et al: Randomized trial of surgery versus surgery followed by adjuvant hepatic arterial infusion with 5 -fluorouracil and folinic acid for liver metastases of colorectal cancer. Ann Surg 228:756-762, 1998

12. D'Angelica MI, Correa-Gallego C, Paty PB, et al: Phase II trial of hepatic artery infusional and systemic chemotherapy for patients with unresectable hepatic metastases from colorectal cancer: Conversion to resection and long-term outcomes. Ann Surg 261:353-360, 2015

13. Kemeny $N$, Capanu $M, D^{\prime}$ Angelica $M$, et al: Phase I trial of adjuvant hepatic arterial infusion (HAl) with floxuridine (FUDR) and dexamethasone plus systemic oxaliplatin, 5-fluorouracil and leucovorin in patients with resected liver metastases from colorectal cancer. Ann Oncol 20:1236-1241, 2009

14. Kemeny N, Jarnagin W, Paty $P$, et al: Phase I trial of systemic oxaliplatin combination chemotherapy with hepatic arterial infusion in patients with unresectable liver metastases from colorectal cancer. J Clin Oncol 23:4888-4896, 2005

15. Kemeny NE, Melendez FD, Capanu M, et al: Conversion to resectability using hepatic artery infusion plus systemic chemotherapy for the treatment of unresectable liver metastases from colorectal carcinoma. J Clin Oncol 27:3465-3471, 2009

16. Kemeny NE, Niedzwiecki D, Hollis DR, et al: Hepatic arterial infusion versus systemic therapy for hepatic metastases from colorectal cancer: A randomized trial of efficacy, quality of life, and molecular markers (CALGB 9481). J Clin Oncol 24:1395-1403, 2006

17. Edge S, Byrd DR, Compton CC, et al: AJCC Cancer Staging Manual (ed 7). New York, NY, Springer, 2010

18. Fong $Y$, Fortner $J$, Sun $R L$, et al: Clinical score for predicting recurrence after hepatic resection for metastatic colorectal cancer: Analysis of 1001 consecutive cases. Ann Surg 230:309-318, 1999; discussion 318-321

19. Bennett JJ, Schmidt CR, Klimstra DS, et al: Perihepatic lymph node micrometastases impact outcome after partial hepatectomy for colorectal metastases. Ann Surg Oncol 15:1130-1136, 2008

20. Cardona K, Donataccio D, Kingham TP, et al: Treatment of extensive metastatic colorectal cancer to the liver with systemic and hepatic arterial infusion chemotherapy and two-stage hepatic resection: The role of salvage therapy for recurrent disease. Ann Surg Oncol 21:815-821, 2014

21. Robins JM, Mark SD, Newey WK: Estimating exposure effects by modelling the expectation of exposure conditional on confounders. Biometrics 48:479-495, 1992

22. Kalbfleisch JD, Prentice RL: The Statistical Analysis of Failure Time Data (ed 2). Hoboken, NJ, Wiley-Interscience, 2002

23. House MG, Kemeny NE, Gönen $M$, et al: Comparison of adjuvant systemic chemotherapy with or without hepatic arterial infusional chemotherapy after hepatic resection for metastatic colorectal cancer. Ann Surg 254:851-856, 2011

24. Hamady ZZ, Lodge JP, Welsh FK, et al: Onemillimeter cancer-free margin is curative for colorectal liver metastases: A propensity score casematch approach. Ann Surg 259:543-548, 2014

25. Deschamps F, Rao P, Teriitehau C, et al: Percutaneous femoral implantation of an arterial port catheter for intraarterial chemotherapy: Feasibility and predictive factors of long-term functionality. J Vasc Interv Radiol 21:1681-1688, 2010

26. Goéré D, Benhaim L, Bonnet $S$, et al: Adjuvant chemotherapy after resection of colorectal liver metastases in patients at high risk of hepatic recurrence: A comparative study between hepatic arterial infusion of oxaliplatin and modern systemic chemotherapy. Ann Surg 257:114-120, 2013

27. Allen PJ, Nissan A, Picon Al, et al: Technical complications and durability of hepatic artery infusion pumps for unresectable colorectal liver metastases: An institutional experience of 544 consecutive cases. J Am Coll Surg 201:57-65, 2005

28. Ito $K$, Ito $H$, Kemeny $N E$, et al: Biliary sclerosis after hepatic arterial infusion pump chemotherapy for patients with colorectal cancer liver metastasis: Incidence, clinical features, and risk factors. Ann Surg Oncol 19:1609-1617, 2012

29. Moulton CA, Gu CS, Law CH, et al: Effect of PET before liver resection on surgical management for colorectal adenocarcinoma metastases: A randomized clinical trial. JAMA 311:1863-1869, 2014

\section{Affiliations}

Bas Groot Koerkamp, Eran Sadot, Nancy E. Kemeny, Mithat Gönen, Julie N. Leal, Peter J. Allen, Andrea Cercek, Ronald P. DeMatteo, T. Peter Kingham, William R. Jarnagin, and Michael I. D’Angelica, Memorial Sloan Kettering Cancer Center, New York, NY; Bas Groot Koerkamp, Erasmus MC Cancer Institute, Rotterdam, the Netherlands; and Eran Sadot, Rabin Medical Center, Petah Tikva, and Tel Aviv University, Tel Aviv, Israel.

\section{Support}

Supported by the Dutch Cancer Society (B.G.K.) and the National Cancer Institute Cancer Center Support Grant No. P30 CA008748.

Prior Presentation

Presented at the annual meeting of the International Hepato-Pancreato-Biliary Association, Sao Paulo, Brazil, April 20-23, 2016. 


\section{AUTHORS' DISCLOSURES OF POTENTIAL CONFLICTS OF INTEREST}

Perioperative Hepatic Arterial Infusion Pump Chemotherapy Is Associated With Longer Survival After Resection of Colorectal Liver Metastases: A Propensity Score Analysis

The following represents disclosure information provided by authors of this manuscript. All relationships are considered compensated. Relationships are self-held unless noted. I = Immediate Family Member, Inst = My Institution. Relationships may not relate to the subject matter of this manuscript. For more information about ASCO's conflict of interest policy, please refer to www.asco.org/rwc or ascopubs.org/jco/site/ifc.

\section{Bas Groot Koerkamp}

No relationship to disclose

\section{Eran Sadot}

No relationship to disclose

\section{Nancy E. Kemeny}

Research Funding: Amgen (Inst)

\section{Mithat Gönen}

No relationship to disclose

Julie N. Leal

No relationship to disclose

Peter J. Allen

No relationship to disclose

\section{Andrea Cercek}

No relationship to disclose

\section{Ronald P. DeMatteo}

No relationship to disclose

\section{T. Peter Kingham}

No relationship to disclose

William R. Jarnagin

No relationship to disclose

Michael I. D'Angelica

No relationship to disclose 


\section{Acknowledgment}

We thank Taryn Boucher for helping with data collection.

\section{Appendix}

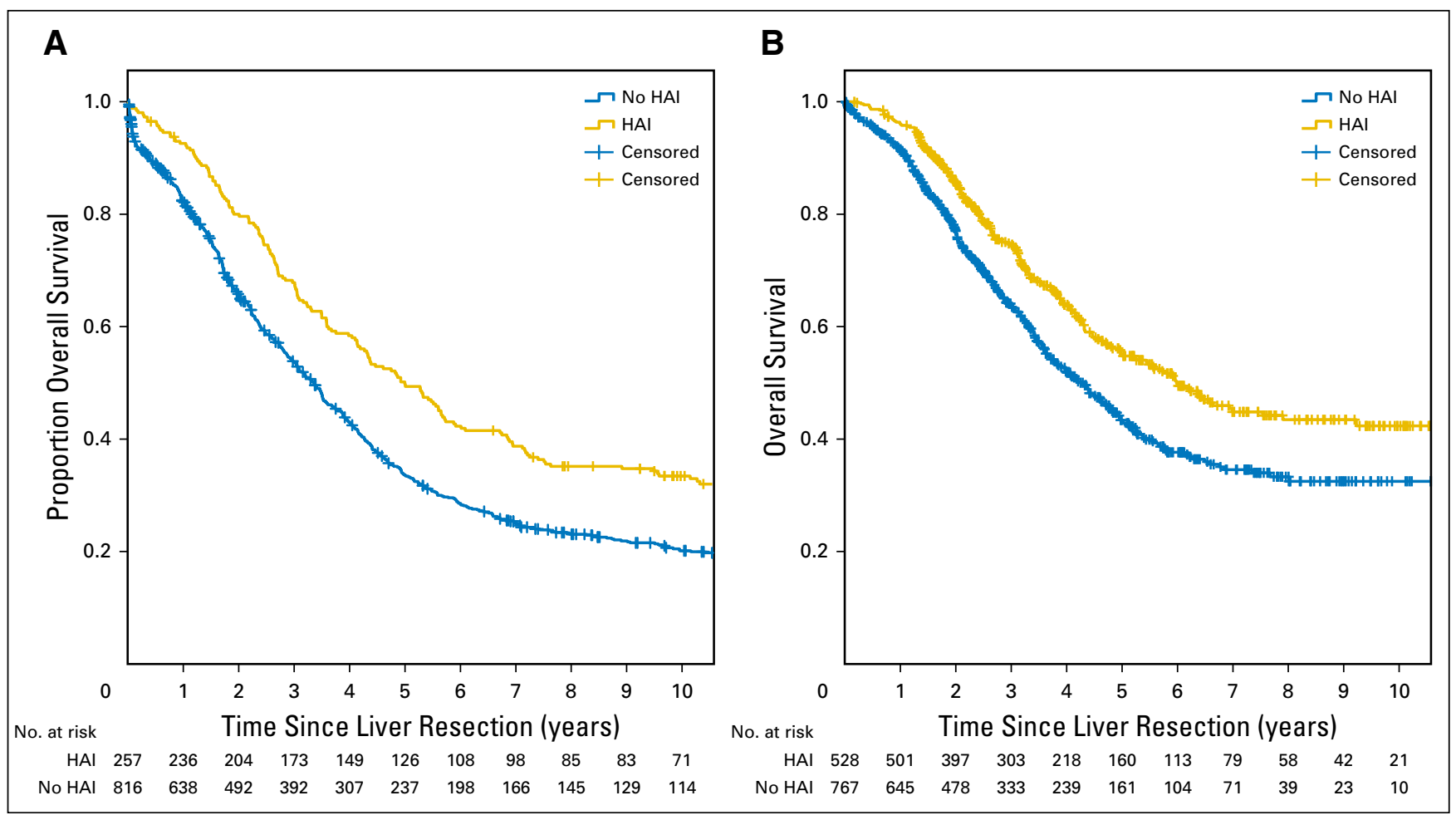

Fig A1. (A) All patients underwent a curative-intent resection of colorectal liver metastases between 1991 and 2002. The median overall survival was 60 months with hepatic arterial infusion pump chemotherapy (HAl) and 40 months without HAI $(P<.001)$. (B) All patients underwent a curative-intent resection of colorectal liver metastases between 2003 and 2012. The median overall survival was 72 months with HAl and 51 months without HAl $(P<.001)$.

\begin{tabular}{|c|c|c|c|c|c|c|c|}
\hline Propensity for HAI & $\mathrm{HAl}$ & Sample Size & Median OS (months) & $95 \% \mathrm{Cl}$ & HR & $95 \% \mathrm{Cl}$ & $P$ \\
\hline Low & $\begin{array}{l}\text { No } \\
\text { Yes }\end{array}$ & $\begin{array}{r}507 \\
76\end{array}$ & $\begin{array}{l}42 \\
86\end{array}$ & $\begin{array}{l}37 \text { to } 47 \\
67 \text { to } 131\end{array}$ & 0.51 & 0.38 to 0.68 & $<.001$ \\
\hline Low-medium & $\begin{array}{l}\text { No } \\
\text { Yes }\end{array}$ & $\begin{array}{l}457 \\
126\end{array}$ & $\begin{array}{l}45 \\
60\end{array}$ & $\begin{array}{l}41 \text { to } 51 \\
52 \text { to } 90\end{array}$ & 0.67 & 0.51 to 0.87 & .002 \\
\hline Medium-high & $\begin{array}{l}\text { No } \\
\text { Yes }\end{array}$ & $\begin{array}{l}353 \\
230\end{array}$ & $\begin{array}{l}49 \\
67\end{array}$ & $\begin{array}{l}41 \text { to } 57 \\
54 \text { to } 90\end{array}$ & 0.72 & 0.57 to 0.91 & .005 \\
\hline High & $\begin{array}{l}\text { No } \\
\text { Yes }\end{array}$ & $\begin{array}{l}244 \\
339\end{array}$ & $\begin{array}{l}48 \\
64\end{array}$ & $\begin{array}{l}41 \text { to } 64 \\
52 \text { to } 79\end{array}$ & 0.77 & 0.61 to 0.98 & .037 \\
\hline
\end{tabular}

NOTE. For the propensity score we used 2,332 patients (98.5\%) who had complete data on all covariates.

Abbreviations: HAl, hepatic arterial infusion pump chemotherapy; HR, hazard ratio; OS, overall survival. 